\title{
Triagem fitoquímica e avaliação da atividade antibacteriana de extratos das flores de Sambucus nigra L. (Caprifoliaceae)
}

MATTE, A.K.'; DEAK, A.R.'; MATA, P.T.G. ${ }^{*}$

${ }^{1}$ Pontifícia Universidade Católica do Paraná. Escola de Saúde e Biociências. Curso de Farmácia. Avenida da União, $n^{\circ}$ 500, Jardim Coopagro, Toledo-PR, CEP: 85902-532. *Autor para correspondencia: patricia.ghezzi@ pucpr.br

RESUMO: O presente trabalho teve como objetivo realizar a triagem fitoquímica e avaliar a atividade antibacteriana de extratos das flores de Sambucus nigra L. Os extratos; aquoso (10\%), etanólico (5\%) e Acetato de etila (5\%) foram submetidos a testes colorimétricos para triagem fitoquímica e a avaliação da atividade antibacteriana foi realizada pelo método de disco-difusão em ágar. Os resultados mostraram que nas concentrações de 6 e $12 \mathrm{mg}$ o extrato aquoso apresentou halos significativos de inibição para Staphylococcus aureus, Pseudomonas aeruginosa e Streptococcus pyogenes, porém, quando comparado aos medicamentos usados como referência a atividade não foi satisfatória, e, ainda, evidenciou a ausência de inibição para todas as cepas testadas com o aumento da concentração para 18 e $24 \mathrm{mg}$. A análise da triagem fitoquímica evidenciou a presença de flavonoides com intensa reação de cor no extrato aquoso e etanólico, e de fraca intensidade no extrato acetato de etila. Nos mesmos extratos, pelos testes realizados, não foram detectados taninos, saponinas, antraquinonas e alcaloides. Concluiu-se que o extrato aquoso apresentou melhor efeito inibitório para Staphylococcus aureus, Pseudomonas aeruginosa e Streptococcus pyogenes, porém insuficiente para promover a inativação eficiente quando comparado aos controles.

Palavras-chave: Sambucus nigra L., atividade antimicrobiana, Caprifoliaceae, plantas medicinais.

\begin{abstract}
Phytochemical screening and evaluation of the antibacterial activity of Sambucus nigra L. flower extracts (Caprifoliaceae). This study aimed to perform a phytochemical screening and to evaluate the antibacterial activity of the extracts of Sambucus nigra $\mathrm{L}$. flowers. The aqueous $(10 \%)$, ethanolic $(5 \%)$ and ethyl acetate $(5 \%)$ extracts were subjected to colorimetric tests for phytochemical screening and the antibacterial activity evaluation was performed by the disk-diffusion method in agar. The results showed that in the 6 and $12 \mathrm{mg}$ concentrations the aqueous extract presented significant inhibition halos for Staphylococcus aureus, Pseudomonas aeruginosa and Streptococcus pyogenes, but when compared with the medicines used as a reference, the activity was not satisfactory and, besides, it indicated the absence of inhibition for all the tested strains with the concentration increase of 18 and $24 \mathrm{mg}$. The phytochemical screening analysis showed the presence of flavonoids with intense color reaction in the aqueous and ethanol extracts, and of low intensity in the ethyl acetate sample. In the same extracts, the tests did not detect tannins, saponins, alkaloids and anthraquinones. It was concluded that the aqueous extract showed better inhibitory effect on the Staphylococcus aureus, Pseudomonas aeruginosa and Streptococcus pyogenes, but not enough to promote an effective inactivation when compared with the medicine tests.
\end{abstract}

Keywords: Sambucus nigra L., antimicrobial activity, Caprifoliacea, medicinal plants.

\section{INTRODUÇÃO}

O uso indiscriminado e prolongado de antimicrobianos tem levado a seleção de microrganismos patogênicos mutantes resistentes às medicações (Vargas et al., 2004), gerando um sério problema de saúde pública (Duarte et al., 2004). Fato esse, abre caminho para pesquisas que 
objetivem identificar novos agentes antimicrobianos (Antunes et al., 2006; Guimarães et al., 2010).

$\mathrm{O}$ uso de plantas medicinais para o tratamento, prevenção e cura de enfermidades acompanha o homem desde as civilizações mais antigas (Firmo et al., 2011). A presença de importantes metabólitos secundários e a identificação de compostos biologicamente ativos (Duarte et al., 2004; Calixto, 2005), faz das plantas medicinais uma alternativa muitas vezes eficaz para o desenvolvimento de novas estratégias terapêuticas para o tratamento de doenças infecciosas (Ming, 1998; Vargas et al., 2004; Loguercio et al., 2005; Calixto, 2005; Carvalho et al., 2014).

Entre os anos de 1981 e 2002, das 877 novas moléculas introduzidas no mercado farmacêutico, $49 \%$ eram substâncias isoladas a partir de produtos naturais (Newman et al., 2003). No ano de 2010, os fitoterápicos representavam aproximadamente $15 \%$ do capital da indústria farmacêutica mundial (Niero, 2010).

Sambucus nigra L. é uma planta arbustiva de origem europeia cultivada no sul do Brasil, e que possui propriedades semelhantes à espécie Sambucus australis (Cham. et Schlecht.). Há registro de seu uso na Idade da Pedra, por gregos e romanos, sendo cultivada até os dias atuais (Lorenzi \& Matos, 2008). Seus frutos, folhas, casca, entrecasca e raízes são amplamente utilizados na medicina tradicional em razão de suas propriedades terapêuticas (diurética, antipirética, anti-séptica, cicatrizante, anti-inflamatória e contra problemas respiratórios) identificadas (Lorenzi \& Matos, 2008; Guarrera, 1999). A infusão das flores é utilizada para diminuir inchaço e irritações oculares, diminuir desconfortos gastrointestinais, estimular a secreção brônquica, no tratamento da tosse e asma, além de possuir ação diurética (Guarrera, 2005). Sabese ainda, que estudos realizados com extratos da entrecasca e raízes dessa planta, evidenciaram uma importante atividade antiviral para o vírus Influenza humano tipo A e tipo B (Zakay-Rones et al., 1995) e para o vírus da imunodeficiência felina, um vírus de escolha para estudos que visam a análise da patogênese, desenvolvimento de vacinas e alternativas de tratamento para o vírus da imunodeficiência humana, respectivamente (Manganelli et al., 2005).

Assim como para avaliação da atividade antiviral, estudos que avaliam a possível atividade antimicrobiana frente a microrganismos de grande importância clínica dessa planta são escassos (Hearst et al., 2010), fato que justifica a proposta dessa pesquisa de avaliar a atividade antibacteriana e realizar a triagem fitoquímica do extrato aquoso (EA), extrato etanólico (EE) e extrato acetato de etila (EAc) das flores de Sambucus nigra L.

\section{MATERIAL E MÉTODO}

As flores de Sambucus nigra L. foram adquiridas no estado seco no comércio de Toledo, Paraná, bem como o laudo técnico da identificação botânica e controle de qualidade do material vegetal, (Lote SABU010712, Data de fabricação 01.05.12 e validade de 3 anos). Foram trituradas até obtenção de um fino pó para então serem conservadas em frasco hermeticamente fechado e ao abrigo da luz.

O Extrato aquoso (EA) a $10 \%$ foi preparado pelo infuso com o pó das flores em água deionizada (Freitas \& Bacchi, 1992) e o extrato etanólico (EE) a $5 \%$ e o extrato acetato de etila (EAc) a $5 \%$ pela maceração das flores em pó nos respectivos solventes (Freitas \& Bacchi, 1992).

A triagem fitoquímica foi realizada seguindo os métodos colorimétricos descritos por Goyal et al., (2010) para a observação de taninos, saponinas, flavonoides, antraquinonas e alcaloides.

O teste de avaliação de atividade antibacteriana foi realizado pelo método de discodifusão em ágar (Bauer et al., 1966). Para os testes de disco-difusão, discos de papel filtro (discos testes) de seis $\mathrm{mm}$ de diâmetro, foram esterilizados em autoclave, e individualmente impregnados com 10 e $20 \mu \mathrm{l}$ dos extratos nas concentrações de 6 e 12 mg respectivamente, e levados para a estufa por 2 horas a $37^{\circ} \mathrm{C}$ para evaporação total dos solventes. Os mesmos volumes de Etanol PA e Acetato de etila PA foram acrescidos em discos de papel filtro para o preparo dos controles do EE e EAc respectivamente, e de água deionizada esterilizada para os controles negativos. Discos testes adicionais de EA foram impregnados com 30 e $40 \mu$ do extrato nas concentrações 18 e $24 \mathrm{mg}$, e levados para a estufa por 2 horas a $37^{\circ} \mathrm{C}$ para evaporação total do solvente.

Os discos testes foram analisados frente a cepas padronizadas de Staphylococcus aureus (ATCC 25922), Escherichia coli (ATCC 25923), Salmonella typhimurium (NEWP 0015), Pseudomonas aeruginosa (ATCC 27853) e Streptococcus pyogenes (NEWP 0015). As cepas foram semeadas em ágar Mueller-Hinton e incubadas a $37^{\circ} \mathrm{C}$ por 24 horas em estufa bacteriológica. A determinação da concentração de microrganismos inoculados por placa foi realizada por análise de turbidez, pela comparação da suspensão preparada (1 mL em solução salina estéril 0,9\% + cepas), com a escala nefelométrica de MacFarland 0,5\% (1,5X $10^{6} \mathrm{UFC} / \mathrm{mL}$ ) (Bauer et al., 1966; NCCLS, 2003).

Medicamentos referências; amoxicilina (10 $\mathrm{mcg}$ ), vancomicina (30 mcg), tetraciclina (30 mcg), ceftriaxona $(30 \mathrm{mcg})$ e penicilina $\mathrm{G}(10 \mathrm{mcg})$ foram utilizados como padrões de interpretação para cada microrganismo testado e a água deionizada estéril foi o controle negativo.

Rev. Bras. PI. Med., Campinas, v.17, n.4, supl. III, p.1049-1054, 2015. 
Após o período de incubação, os diâmetros dos halos de inibição para cada concentração foram interpretados de acordo com os critérios de interpretação preconizados pelo NCCLS (National Committee for Clinical Laboratory Standards, 1997. 2000 a e 2000b). Foi classificado como positivo, o extrato que apresentou halo de inibição maior ou igual a 9 mm (Smânia et al., 1995).

Todos os ensaios foram realizados em triplicata e a avaliação estatística foi realizada com software GraphPad Prism 5 pelo teste ANOVA. Os resultados foram expressos como média \pm desvio padrão da média, e quando $f$ foi significativo, as diferenças entre as médias foram avaliadas com valor de $p$ corrigido para o teste de Tukey. O nível de significância avaliado foi de $p<0.05$.

As análises das variáveis foram tabuladas e transcritas para Microsoft Excel 2010.

\section{RESULTADO E DISCUSSÃO}

A análise da triagem fitoquímica evidenciou a presença de flavonoides; com intensa reação de cor (+++) nos EA e EE, e de fraca intensidade $(+)$ no EAc (Tabela 1). Esse resultado confirma a informação fornecida no laudo de identificação da espécie comercializada e de estudos que afirmam a presença de flavonoides $(>3,0 \%)$, especialmente a rutina $(1,5 \%$ a $3,5 \%)$ e quercetina $(1,51 \%)$ como principais constituintes das flores de Sambucus nigra L. (Charaux, 1924; Davídek, 1961; Shoaib et al., 1972; Newall et al., 2002; Veberic et al., 2009; Hearst et al., 2010).

Flavonoides estão associados às ações biológicas ativas e sua presença em plantas está diretamente relacionada à significativa atividade antimicrobiana (Schlemper et al., 1998; Sartori et al., 2003; Andrade et al., 2005; Calixto, 2005), consequência da sua capacidade em formar complexos extracelulares, solubilizar proteínas, e quando mais lipofílicos, penetrar com mais facilidade nas membranas celulares promovendo uma lise microbiana eficaz (Ahmed et al., 2005; Sobrinho, 2013). Nos flavonoides, a afinidade de seus grupos fenólicos hidroxilo por proteínas, permite que atuem como inibidores de enzimas tanto em bactérias Gram - negativas como em Gram- positivas, interferindo nas suas vias de síntese, inibindo seu desenvolvimento (Sato et al., 1996; Alcaráz et al., 2000; Ávila et al., 2008; Sobrinho, 2013; Li et al., 2012; Sato et al., 1996).

Os halos formados no meio de cultura refletem o potencial inibitório do extrato em relação às bactérias testadas; portanto, a atividade antibacteriana do extrato é diretamente proporcional ao diâmetro do halo de inibição. Extratos classificados como muito ativos, apresentam halos inibitórios de treze a dezoito $\mathrm{mm}$, extratos ativos formam halos entre nove e doze $\mathrm{mm}$ e os inativos, halos de nove mm ou menos (Alves et al., 2000). O presente estudo evidenciou que o EA na concentração de $12 \mathrm{mg}$, apresentou atividade antibacteriana classificada como muito ativa, frente as cepas de Staphylococcus aureus, e ativa para Pseudomonas aeruginosa e Streptococcus pyogenes, bactérias invasivas e nocivas ao homem (Trabulsi \& Althertum, 2008). A inibição foi significativa apenas para Staphylococcus aureus $(p<0,001)$ na concentração de $12 \mathrm{mg}$ (Tabela 2).

Com o aumento da concentração do EA, acreditou-se que a atividade antibacteriana se mantivesse estável ou mais evidente, mas com a elevação a atividade inibitória diminuiu, com halos de inibição praticamente ausentes para todos os microrganismos na concentração de $24 \mathrm{mg}$ (Tabela 2). Essa diminuição da atividade biológica pode estar relacionada com o aumento da toxicidade da planta, resultado do aumento da concentração, ou ainda, da interação de seus metabólitos (Brito et. al., 1996; Hearst et al. 2010). Não foram encontrados estudos que avaliem o grau de toxicidade de Sambucus nigra L. O uso indiscriminado, sem ter conhecimento de fato das propriedades cientificamente comprovadas de plantas pode ocasionar alterações graves na saúde do indivíduo (Hostettmann et al., 2003). Estudos mostram que aproximadamente $26 \%$ das plantas consideradas medicinais que passaram por ensaios de toxicidade, tiveram a comprovação dessa atividade, evidenciando que ao invés de promover o bem estar, podem provocar graves danos à saúde

TABELA 1. Triagem fitoquímicado extrato aquoso (EA), extrato etanólico (EE) e extrato acetato de etila (EAc) obtidos das flores de Sambucus nigra L.

\begin{tabular}{lccc}
\hline Compostos & EA & EE & EAc \\
\hline Taninos & - & - & - \\
Flavonoides & +++ & +++ & - \\
Saponinas & - & - & - \\
Antraquinonas & - & - & - \\
Alcaloides & - & - & + \\
\hline
\end{tabular}

Legenda: Intensidade da reação colorimétrica classificada como; reação intensa (+++), moderada (++) fraca (+) e ausente (-). 
TABELA 2. Valores das médias $(\mathrm{mm}) \pm$ Desvio Padrão dos halos inibitórios, do extrato aquoso (EA) nas concentrações de 6, 12, 18 e 24 mg, e do extrato etanólico (EE) e extrato acetato de etila (EAc) nas concentrações de 6 e 12 mg, obtidos das flores de Sambucus nigra L.

\begin{tabular}{|c|c|c|c|c|c|}
\hline Extrato -Concentração & SA & EC & PA & ST & SP \\
\hline EA - $6 \mathrm{mg}$ & $8,0 \pm 0,00$ & $6,0 \pm 0,00$ & $10,0 \pm 0,00$ & $6,7 \pm 1,15$ & $8,7 \pm 1,15$ \\
\hline $\mathrm{EA}-12 \mathrm{mg}$ & $13,3 \pm 3,06^{* * *}$ & $8,0 \pm 0,00$ & $12,0 \pm 0,00$ & $8,7 \pm 1,15$ & $11,3 \pm 1,15$ \\
\hline $\mathrm{EA}-18 \mathrm{mg}$ & $0,0 \pm 0,00$ & $0,3 \pm 0,00$ & $3,3 \pm 0,00$ & $0,0 \pm 0,00$ & $1,3 \pm 0,00$ \\
\hline EA - $24 \mathrm{mg}$ & $0,3 \pm 0,00$ & $0,0 \pm 0,00$ & $0,7 \pm 0,00$ & $0,0 \pm 0,00$ & $0,7 \pm 0,00$ \\
\hline $\mathrm{EE}-6 \mathrm{mg}$ & $4,0 \pm 0,00$ & $2,0 \pm 0,00$ & $2,3 \pm 0,58$ & $0,0 \pm 0,00$ & $4,7 \pm 0,58$ \\
\hline $\mathrm{EE}-12 \mathrm{mg}$ & $5,5 \pm 0,58$ ** & $6,0 \pm 0,00^{\star \star *}$ & $6,3 \pm 0,58^{\star * *}$ & $0,0 \pm 0,00$ & $5,7 \pm 0,58$ \\
\hline $\mathrm{EAc}-6 \mathrm{mg}$ & $0,0 \pm 0,00$ & $0,0 \pm 0,00$ & $0,0 \pm 0,00$ & $0,0 \pm 0,00$ & $0,0 \pm 0,00$ \\
\hline EAc- $12 \mathrm{mg}$ & $0,0 \pm 0,00$ & $0,0 \pm 0,00$ & $0,0 \pm 0,00$ & $0,0 \pm 0,00$ & $0,0 \pm 0,00$ \\
\hline
\end{tabular}

Legenda: Os valores foram expressos em média \pm Desvio Padrão. Diferença significativa de $\left(^{* * *}\right) p<0,001,\left(^{* *}\right) p<0,01$ e $\left(^{*}\right) p<0,05-$ comparado ao extrato de menor concentração. (SA) Staphylococcus aureus, (EC) Escherichia coli, (PA) Pseudomonas aeruginosa, (ST) Salmonella typhimurium e (SP) Streptococccus pyogenes.

de quem faz seu uso indiscriminado (OECD, 2001). Os halos de inibição do EE foram menos significativos quando comparadas aos halos obtidos com o EA para todos os microrganismos testados, mesmo com o aumento da concentração. Pseudomonas aeruginosa, Escherichia coli ( $\mathrm{p}$ $<0,001)$ e Staphylococcus aureus $(p<0,005)$ demonstraram aumento de atividade antibacteriana com o aumento na concentração do EE (Tabela 2), porém com sensibilidade ao extrato classificada como resistente quando comparada aos halos de inibição obtidos com os medicamentos padronizados (Tabela 3). O EAc não inibiu o desenvolvimento de nenhum dos microrganismos testados. Esses resultados podem ser resultantes da dificuldade de difusão dos extratos no meio de cultura (Violante, 2008).

A água é um solvente polar, o acetato de etila possui média polaridade e o etanol é classificado como um solvente bipolar. A solubilidade de uma substância orgânica está diretamente relacionada à estrutura molecular, especialmente com a polaridade das ligações e da espécie química como um todo (Martins et al., 2013). Cocos e bacilos não fermentadores de glicose foram os microrganismos que apresentaram resultados mais expressivos de inibição frente aos extratos testados: Bactérias dos gêneros Staphylococcus sp. e Streptococcus $s p$. são Gram-positivas, ou seja, com parede celular constituída de peptidioglicano (70 a $75 \%$ ), que Ihes confere uma forte carga positiva (Trabulsi \& Althertum, 2008). Pseudomonas aeruginosa é uma bactéria Gram-negativa, que possui parede celular composta por uma dupla camada de lipídica (uma barreira hidrofóbica adicional) sobrepondo a delgada camada de peptidioglicanos, fato que confere a essas bactérias uma permeabilidade parcialmente seletiva regulada por canais proteicos, que tendem a controlar a entrada de substâncias, muitas vezes, pelo seu tamanho (França \& Kuster, 2009; Trabulsi \& Althertum, 2008). Nesse estudo, o solvente utilizado que obteve resultados mais significativos (água)

TABELA 3. Atividade antibacteriana dos medicamentos padronizados ante os microrganismos testados.

\begin{tabular}{cccccc}
\hline & \multicolumn{5}{c}{ Microrganismos } \\
\cline { 2 - 5 } Antibióticos & AS & EC & ST & PA & SP \\
\hline AMO & S 32 & S 18 & S 32 & S 30 & S 32 \\
VAN & R 6 & R 6 & R & R - & R 6 \\
TET & S 28 & I 18 & S 24 & S 30 & S 30 \\
CRO & S 38 & S 24 & S 36 & S 36 & S 40 \\
PEN & R 22 & R 14 & R 16 & R 16 & I 20 \\
CIP & S 38 & S 28 & S 36 & S 38 & S 40
\end{tabular}

Legenda: Antibióticos comerciais: Amoxicilina $10 \mathrm{mcg}$ (AMO), Vancomicina $30 \mathrm{mcg}$ (VAN), Tetraciclina $30 \mathrm{mcg}$ (TET), Ceftriaxona 30 mcg (CRO), Penicilina G $10 \mathrm{mcg}$ (PEN) e Ciprofloxacina $5 \mathrm{mcg}$ (CIP). R= Resistente; I= Intermediário; S= Sensível; - (ausência de halo inibitório). (SA) Staphylococcus aureus, (EC) Escherichia coli, (PA) Pseudomonasaeruginosa, (ST) Salmonellatyphimurium e (SP) Streptococccuspyogenes. 
é polar, o que pode ter favorecido o processo de solubilização de substâncias químicas presentes nas flores de Sambucus nigra L., resultando em uma atividade antibacteriana mais expressiva do EA, e menos significativa e ausente para o EE e EAc, respectivamente, frente aos microrganismos testados.

\section{CONCLUSÕES}

A análise da triagem fitoquímica evidenciou a presença de flavonoides com intensa reação de cor para oEAeEE e de fraca intensidade para oEAc, além da ausência de taninos, saponinas, antraquinonas e alcaloides em todos os extratos testados. O EA apresentou considerável ação antibacteriana frente bactérias de grande importância clínica, mesmo que limitada. Os resultados encontrados no estudo confirmam o uso etnomedicinal com finalidade antibacteriana das flores de Sambucus nigra $\mathrm{L}$

\section{REFERÊNCIAS}

AHMED, F. et al., Antibacterial activity of Ludwigia adscendens. Fitoterapia, v.76, n.5, p.473-75, 2005.

ALCARÁZ, L.E. et al. Antibacterial Activity of Flavonoids Against Methicilin - resistant Staphylococcus aureus strains. Journal of Theoretical Biology, v.205, n.2, p. $231-40,2000$.

ALVES, T.M.A. et al. Biological Screening of Brazilian Medicinal Plants. Memórias do Instituto Oswaldo Cruz, v.95, n.3, p.367-73, 2000.

ANDRADE, C.A. et al. Avaliação da atividade antibacteriana e triagem fitoquímica das flores de Acacia podalyriifolia A. Cunn. ex. G. Don Leguminosae-Mimosoidea. Revista Brasileira Farmacognosia, v.15, n.1, p.13-15, 2005.

ANTUNES, R.M.P. et al. Atividade antimicrobiana "in vitro" e determinação daconcentração inibitória mínina (CIM) de fitoconstituintes e produtossintéticos sobre bactérias e fungos leveduriformes. Revista Brasileira de Farmacognosia, v.16, n.4, p.517-24, 2006.

ÁVILA, P.H. et al. Structure - activity relationship of antibacterial chalcones. Bioorganic and Medicinal Chemistry, v. 16, n.2, p.9790 - 94, 2008.

BAUER, A. W. et al. Antibiotic susceptibility testing by a standardized single disc method. American Journal of Clinical Pathology. v.45, n.4, p.493-96, 1966.

BRITO, A.R.M.S. Legislação de fitoterapicos. In: STASI, L.D. Plantas medicinais:arte e ciência. Um guia de estudo interdisciplinar, São Paulo: UNESP, p.187-97,1996.

CALIXTO, J.B. Efficacy, safety, quality control, marketing and regulatory guidelines for herbal medicines (phythoterapeutic agents). Brazilian Journal of Medical and Biological Research, v.33, n.2, p.17989, 2005.

CARVALHO, A.F. et al. Avaliação da atividade antibacteriana de extratos etanólico e de ciclohexano a partir das flores de camomila (Matricaria chamomilla L.). Revista Brasileira de Plantas Medicinais, v.16, n.3, p.521-26, 2014.

CHARAUX, M.C. Sur La présence de la rutine dans certains védétaux. Preparation et identification de ce et de sés produits de dédoublèment. Bulletin de la Societé de Chimie Biologique, v.6, p.641-47, 1924.

DAVÍDEK, J. Isolation of chromatographically pure rutin from flowers of Elder. Nature, v.189, n.4763, p.487488, 1961.

DUARTE, M.C.T. et al. Atividade antimicrobiana de extratos hidroalcóolicos de espécies da coleção de plantas medicinais CPQBA/UNICAMP. Revista Brasileira de Farmacognosia, v.14, n.1, p.06-08, 2004.

FIRMO, W.D.C.A. et al. Contexto histórico, uso popular e concepção científica sobre plantas medicinais. Caderno de pesquisa, v.18, n.especial, 2011.

FRANÇA, H.S.; KUSTER, R.M. Atividade antibacteriana de floroglucinóis e de extrato hexânico deHypericum brasiliense Choysi. Química Nova, v.32, n.5,p.1103-1106, 2009.

FREITAS, P.C.D.; BACCHI, E.M. Práticas de Farmacognosia. 3.ed. São Paulo. FCF/USP, 1992.

GUARRERA, P.M. Traditional antihelmintic, antiparasitic and repelenteuses of plants in central Italy. Journal of Ethnopharmacology, v.68, n1-3, p. 183-92, 1999.

GUARRERA, P.M. Traditional phytotherapy in Central Italy (Marche, Abruzzo, and Latium). Fitoterapia, v. 76, n.1, p. 1-25, 2005

GOYAL, A. K. et al. Evaluation of the DPPH radical scavenging activity, total phenols and antioxidant activities in Indian wild Bambusa vulgaris "Vittata" methanolic leaf extract. Journal of Natural Pharmaceuticals, v. $1, n$. 1, p. 40-45, 2010.

GUIMARÃES, D.O. et al. Antibióticos: importância terapêutica e perspectivas para a descoberta e desenvolvimento de novos agentes. Química Nova, v. 33, n. 3, p. 667-79, 2010.

HEARST et. al. Antibacterial activity of Elder (Sambucusnigra L.) flower or berry against hospital pathogens. Journal of Medicinal Plants Research, v. 4, n. 17, p. 1805-09, 2010.

HOSTETTMANN, K. et al. A importância das plantas medicinais: Princípios ativos de plantas superiores. Série de textos da Escola de Verão em Química- IV, São Carlos, SP, EdUFSCar, 2003, 152 p.

LI, Y. et al. Design, synthesis and antimicrobial activities of nitroimidazolederivaties containing 1,3,4-oxadiazole scaffold as FabH inhibitors. Bioorganic and Medicinal Chemistry, v. 20, n.14, p. 4316-22, 2012.

LOGUERCIO, A.P. et. al. Atividade antibacteriana de extrato hidro-alcoólico de folhas de jambolão (Syzygiumcumini(L.) Skells), Ciência Rural, v.35, n.2, p.371-376, 2005.

LORENZI, H.; MATOS, F. J. A. Plantas medicinais no Brasil - nativas e exóticas. 2.ed. São Paulo: Instituto Plantarum, 2008, 512p.

MANGANELLI, R.E.U. et al. Antiviral activity in vitro of Urtica dioica L. Parietaria diffusa M. et K. and Sambucus nigra L. Journal of Ethnopharmacology, v. 98, n.3, p. 323-27, 2005.

MARTINS, C.R. et al. Solubilidade das substâncias orgânicas. Química Nova, v.36, n.8, p.1248-55, 2013.

MING, L.C. Plantas Medicinais, aromáticas e condimentares - avanços na pesquisa agronômica. 
v.2, Botucatu: Unesp, 1998.169p.

WAYNE, P.A. National Committee for Clinical Laboratory Standards. Approved standard M2-A7: Performance standards for antimicrobial disk susceptibility tests, 1997.

WAYNE, P.A. National Committee for Clinical Laboratory Standards. Approved standard M7-A5: Methods for dilution antimicrobial susceptibility test for bacteria that grow aerobicaly, 2000a.

WAYNE, P.A. National Committee for Clinical Laboratory Standards. Performance standards for antimicrobial susceptibility testing; tenth informational supplement M100-S10, 2000b.

WAYNE, P.A. National Committee for Clinical Laboratory Standards. Methods for Dilution Antimicrobial Susceptibility Tests for Bacteria That Grow Aerobically; Approved Standard, 2003.

NEWALL, C.A; ANDERSON, LA; PHILLIPSON, JD. Plantas Medicinais: Guia para profissional de Saúde. 1ed. São Paulo: Editora Premier, 2002. 123p.

NEWMAN, D. J. Natural products as sources of new drugs over the period 1981-2002. Journal of Natural Products, v.6, n.7,p.1022-1037, 2003.

NIERO, R. Fármacos, Fitofármacos e Fitoterápicos: Abordagem Econômica e de Mercado. In: BRESOLIN, T.M.; CECHINEL-FILHO, V. (Org.). Fármacos e Medicamentos: uma abordagem multidisciplinar. 1ed. São Paulo: Editora Santos, 2010. 65p.

OECD (Organization for Economic Co-operation and Development). OECD Guidelines for the Testing of Chemicals. Guideline 423 Acute Oral Toxicity-Acute Toxic Class Method. Paris: OECD. 2001. 20p.

SARTORI, M.R.K. et al. Antifungal activity of fractrions and two pure compounds of flowers from Wedelia paludosa (Acmelabrasiliensis) (Astearaceae). Pharmazie, v.58, n.8, p.567-69, 2003.

SATO, M. et al. Antibacterial activity of hydroxychalcone against methicilin -resistance Staphylococcus aureus. International Journal of Antimicrobial Agents, v. 6, p. 227-31, 1996.

SOBRINHO, C.R.W. Determinação da atividade antimicrobiana e citotóxica de extratos da casca e do caule Anadenathera colubrina (Vell.) Brenan var. Cebil(Griseb.) Von Reis Alt. (angico-de-caroço). 2013, 120p. Dissertação (Mestrado em Ciências Farmacêuticas) - Instituto de Ciências da Saúde, Universidade Federal do Pará, Belém-PA.

SCHLEMPER, S.E.M. Atividade antibacteriana de frações semipurificadas e compostos puros de Wedelia paludosa (Compositae). 1998. 81p. Dissertação (Mestrado em Ciências Farmacêuticas) Universidade do Vale do Itajaí, Itajaí, SC.

VEBERIC, R. et al. European elderberry (Sambucus nigra L.) rich in sugar, organic acidis, anthocyanins and selected polyphenols. Food Chemistry, v. 114, n.2, p. 511-15, 2009.

SHOAIB, A. M. et al. A note on flavonoid and cargohydratecontents of Sambucusnigra L. grown in Egypt. Journal of Pharmaceutical Sciences, v.13, n.1, p. 95-100, 1972.

SMÂNIA, A. et al. Antibacterial activity of a substance produced by the fungus Pycnoporus sanguineus (Fr.) Murr. Journal of Ethnopharmacology, v.45, n.3, p.177-81, 1995.

TRABULSI, L.R.; ALTERTHUM, F. Microbiologia. 5ed. São Paulo: Atheneu, 2008. 760p.

VARGAS, A.C. et al.Atividade antimicrobiana "in vitro" de extrato alcóolico de própolis, Ciência Rural, v.34, n.1, p.159-163, 2004.

VIOLANTE, I.M.P. Avaliação do potencial antimicrobiano e citotóxico de espécies vegetais do Cerrado da Região Centro-Oeste. 2008. 72p. Dissertação (Mestrado em Ciências Farmacêuticas - Saúde e Desenvolvimento na Região Centro-Oeste) Universidade Federal de Mato Grosso do Sul, Campo Grande.

ZAKAY-RONES, Z. et al. Inhibition of Several Strains of Influenza Virus in Vitro and Reduction of Symptoms by an Elderberry Extract (Sambucus nigra L.) during an Outbreak of Influenza B Panama. The Journal of Alternative and Complementary Medicine. v.1, n.4, p.361-69, 1995. 